\title{
Identifying the determinants of use of the G\&G interventions for older adults in health and social care: protocol of a multilevel approach
}

Daphne Kuiper ${ }^{1 *}$, Martine M Goedendorp ${ }^{1}$, Robbert Sanderman ${ }^{1,2}$, Sijmen A Reijneveld ${ }^{1}$ and Nardi Steverink ${ }^{1,3}$

\begin{abstract}
Background: Despite aging-related losses, many older adults are able to maintain high levels of subjective wellbeing. However, not all older adults are able to self-manage and adapt. The GRIP\&GLEAM [Dutch: GRIP\&GLANS] (G\&G) interventions have shown to significantly improve self-management ability, well-being and loneliness in older adults. Actual use of the evidence-based G\&G interventions, however, remains limited as long as the interplay between implementation factors at different hierarchical stakeholder levels is poorly understood. The aim of the study is to identify the determinants of successful implementation of the G\&G interventions.

Methods/design: The study is performed in health and social care organizations in the northern part of the Netherlands. The degree of implementation success is operationalized by four parameters: use (yes/no), pace (time to initial use), performance (extent of use) and prolongation (intention to continue use). Based on the Fleuren model, factors at four hierarchical stakeholder levels (i.e. target group, professionals, organizations and financial-political context) are assessed at three measurement points in 2 years. The nested data are analyzed applying multilevel modeling techniques.

Discussion: In this study, health and social care organizations are considered to be part of multilevel functional systems, in which factors at different hierarchical stakeholder levels impede or facilitate use of the G\&G interventions. Strengths of the study are the multifaceted measurement of use, and the multilevel approach in identifying the determinants. The study will contribute to the development of ecologically valid implementation strategies of the G\&G interventions and comparable evidence-based practices.
\end{abstract}

Keywords: Older adults, Self-management ability, Well-being, Loneliness, Evidence-based, Use, Implementation, Multilevel, Health, Social care

\section{Background}

Despite an increase in chronic diseases, people are living longer with less disability and fewer functional limitations [1]. Health, therefore, is recently being redefined into the more dynamic concept of 'the ability to adapt and self-manage in the face of social, physical and emotional challenges' [2]. Many older adults are able to adapt

\footnotetext{
*Correspondence: d.kuiper@umcg.nl

${ }^{1}$ Department of Health Sciences, University Medical Center Groningen,

University of Groningen, Groningen, The Netherlands

Full list of author information is available at the end of the article
}

and self-manage, to maintain high levels of well-being, and to live independently up to very old age. Unfortunately, however, this does not hold for all older adults. Prevalence rates of loneliness [3, 4], social isolation [5], depression [6], and inactivity [7] in community-dwelling older adults are growing. Given the rapid increase in the number of older adults and the accumulation of the negative conditions mentioned, interventions that mitigate these conditions are called for [8].

The GRIP\&GLEAM [Dutch: GRIP\&GLANS] (G\&G) interventions have shown to significantly improve 
self-management ability, well-being, and loneliness in older adults $[9,10]$. Based on a common theoretical concept [11], two interventions have been developed: the G\&G home visits and the G\&G group course. Both interventions have been evaluated in randomized clinical trials $[9,10]$. Positive effects were found on self-management ability, well-being and loneliness. The G\&G interventions are designed for older people who have lost-or are at risk of losing-resources in several domains of functioning, which may lead to a diminished capacity for managing new losses or changes. Moreover, the G\&G interventions are based on an explicitly positive concept: they focus on what individuals are still willing and able to do and not on the problems they are confronted with. The self-management abilities taught are not only intended as a response to loss but also as a tool to be used before loss has occurred. The G\&G interventions are therefore also preventive in nature, aiming at the strengthening of one's generative capacity to self-manage regarding all important aspects of well-being and health simultaneously [11].

Many older adults could benefit from the G\&G interventions when the interventions would be routinely provided in health and social care services. However, the actual use of evidence-based practices (EBPs), such as the G\&G interventions, remains limited in the Netherlands [12] as well as internationally [13, 14]. Despite the increasing availability of, and demand for, well-validated interventions, only about $50 \%$ of the interventions delivered in health care are evidence-based [15]. In social work this percentage appears to be even lower [16]. Moreover, actual use of EBPs is only significant to the extent that these practices are sustained for a longer period of time [17].

Three problems complicate the study of determinants of EBP-use. First, there is a wide array of facilitating and impeding factors affecting the use of EBPs. Systematic reviews produce comprehensive lists, ranging from 23 up to 50 different factors [18-22]. Second, EBP-use is a process, not an event [21]. Generally four stages are discerned: orientation, adoption, implementation (i.e. actual use) and continuation [19]. Empirical evidence which factor is important at what stage is, however, scarce. Third and last, different factors seem to operate on different hierarchical stakeholder levels, such as the individual level, the organizational, and the financial-political level [23]. This makes the study of determinants of EBP-use extra complex and explains why explicit multilevel studies on the determinants of EPB-use are also still scarce.

Historically, the focus of most studies has been on the individual level of the professional who is expected to change his routine in a way that enables the use of the new EBP [24]. Recently, a growing number of studies also encompass factors at the organizational and the financial-political level [25, 26]. A serious problem of these studies is, however, that the design and statistical methods are not fit to capture the complex interplay between phenomena at the several different hierarchical levels [15]. For example, self-efficacy (individual professional level), positive work climate (organizational level), and funding (financial-political context level) have been identified as important facilitating factors to EBP-use [23]. But, as of yet, it is not known which of these factors is decisive with respect to EBP-use in the presence of the other two. Answers to this type of questions can only be found when factors at more than one stakeholder level are assessed simultaneously, and when the nested data are analyzed employing multilevel modeling techniques.

The overall aim of the study is to identify the determinants of successful implementation of the G\&G interventions. The concrete objectives of the study are, first, to describe the variation in actual use between organizations, expressed in terms of pace, performance and prolongation. And, second, to explain this variation at consecutive time points in the process by analyzing the factors at four hierarchical stakeholder levels (i.e. target group, professionals, organizations and financial-political context).

\section{Theoretical framework}

In this study, the delineation of the stages of use, and the possible factors affecting the use of the G\&G interventions, is theoretically informed by the model of Fleuren et al. [19]. In the past decade, a large number of models and frameworks on implementation processes has emerged [19, 20, 27-33]. Most of them acknowledge the multi-stage character (i.e. different consecutive phases) and the multi-level structure (i.e. factors at more than one stakeholder level) of the implementation process. We choose to use the Fleuren model above other models, because it incorporates the multi-level and multi-stage approach of implementation processes and combines it with comprehensiveness and practicality. No other model or framework seemed to give such detailed and clear directions to decide, for each stakeholder level, which factor could be important at what stage in the implementation process [34].

Categorized in four levels, the Fleuren model provides a list of 50 factors. A full description of each factor is given, as well as expectations about the direction of influence of each factor (e.g. 'high staff turnover' impedes and 'low staff turnover' facilitates implementation). This equipped us with adequate detail to prepare the content of the assessments in the consecutive measurement waves. For example, 'formal reinforcement' (a factor at the organizational level) is expected to facilitate the transition from adoption to initial implementation (i.e. the 
start of actual use) and 'observability of effects' (a factor at the professional level) is expected to facilitate the transition from implementation to continuation. Based on the Fleuren model, we were able to decide when to look at which factors and how to operationalize them.

Figure 1 shows the theoretical framework of the study. The process of innovation the organizations are expected to go through is divided into three stages: adoption, implementation and continuation. All possible factors of the Fleuren model are categorized at four stakeholder levels (i.e. target group, professionals, organizations and financial-political context). Moreover, within each stakeholder level, we sorted the factors into theoretically meaningful clusters, such as innovation-related factors,

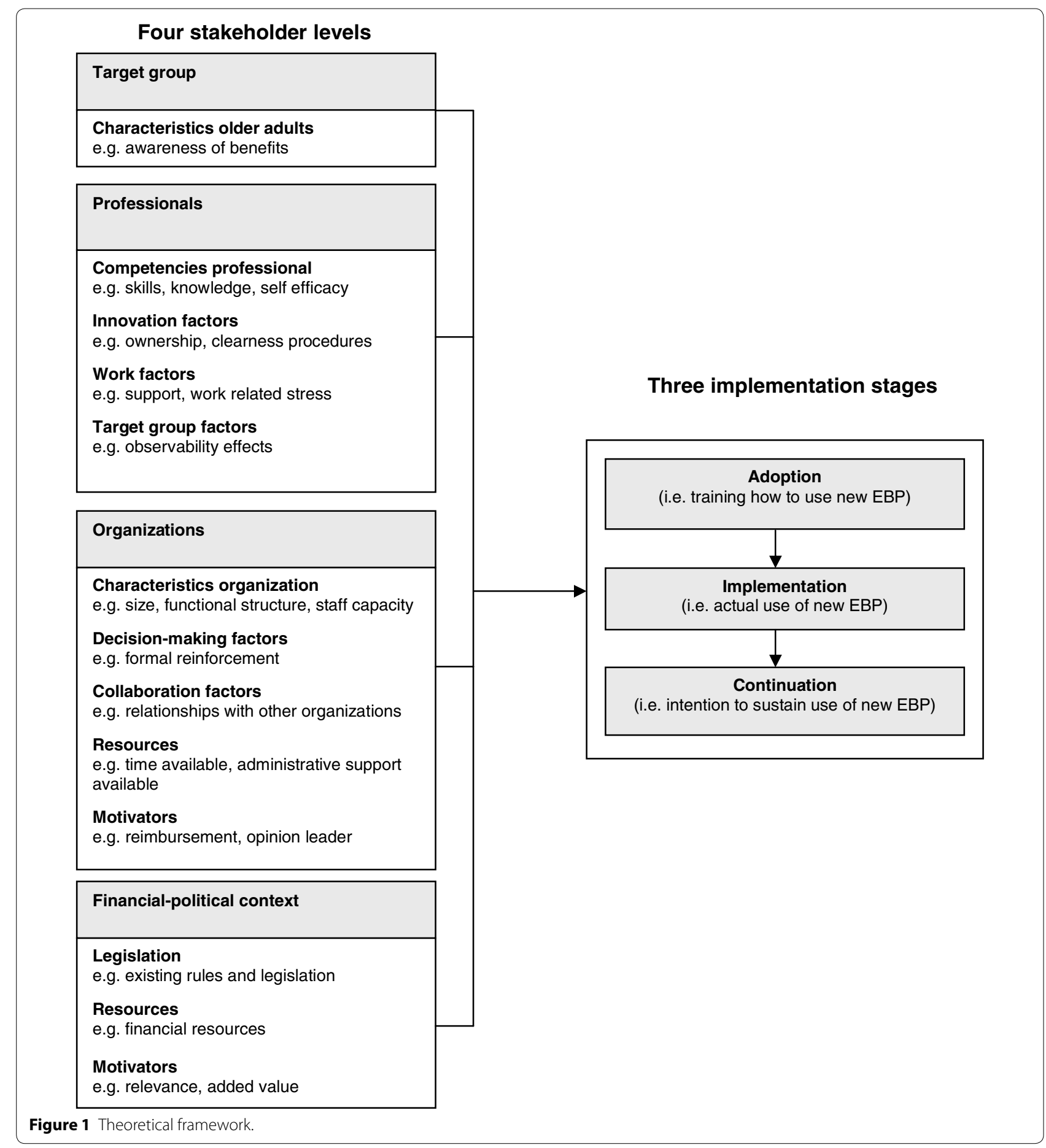


work-related factors, etc. In Additional file 1 the original model of Fleuren is described, as well as the minor adaptations we made.

\section{Methods}

\section{Overview of the project}

The study described in this protocol is part of a larger project that aims to promote and support the use of the evidence-based G\&G interventions in health and social care organizations in the northern part of the Netherlands. Besides identifying the determinants of use of the G\&G interventions, another goal of the larger project is to determine the effectiveness of the G\&G interventions again. However, the study protocol at hand will only describe the former study, i.e. the study on the determinants of successful implementation of the G\&G interventions.

Four partly overlapping phases can be distinguished in the study at hand. In phase 1 the G\&G interventions are disseminated to the field of social and health care organizations. This is being done by means of G\&G workshops, given by the G\&G project team, at strategic meetings where professionals and managers of organizations gather. Because the larger project, of which this study is part, is not a 'top-down' initiative, organizations participate voluntarily. So, any organization that wants to adopt the G\&G-interventions can take part in the project. The goal of phase 1 is to motivate at least 15 organizations to adopt the G\&G interventions and participate in the study.

In phase 2 at least 30 professionals (two per organization) are trained to perform the G\&G interventions. In phase 3 the trained professionals start implementing the G\&G interventions in their organizations by recruiting older adults for participation and, subsequently, delivering the G\&G interventions to them. The core of the empirical study takes place in phase 3 . During that phase, the stages of implementation each organization goes through, are being monitored continuously by the project team, and the facilitating and impeding factors will be assessed at all stakeholder levels in three data collection waves. In phase 4 the data analyses will be executed. A detailed description of the four phases is given in Additional file 2.

The study protocol has been evaluated by the ethics committee of the University Medical Center of Groningen in May 2010. The study was considered to evaluate care as usual and therefore the study was exempted from the Medical Research Involving Human Subjects Act. The study was further performed in accordance with the Helsinki declaration. Informed consent will be given orally.

\section{The interventions}

The two G\&G interventions have the same theoretical basis [11], but are available in two delivery modes: the G\&G home visits and the G\&G group course. Both are considered in the empirical study at hand. The G\&G home visits are delivered by a G\&G coach in six individual home visits of $1.5 \mathrm{~h}$. The G\&G group course is delivered by two G\&G teachers in 6 weekly meetings of $2.5 \mathrm{~h}$ and a booster session after 3 months. The home visits are intended to be delivered to both women and men, aged $\geq 65$ years, who are physically and psycho-socially vulnerable and unable to travel to a group location. The group course is intended to be delivered to a group of around $\mathrm{n}=10$ socially vulnerable women, aged $\geq 55$ years, who subscribe individually, and are physically capable of travelling to a group location. Both G\&G interventions are described in detail in manuals, one for the G\&G coach and one for the G\&G teachers. There is also a workbook for each participant. The content of the G\&G interventions is described in detail elsewhere $[9,10]$.

The training by which professionals become a certified G\&G coach or G\&G teacher involves two and a half days, and is given by master trainers of the G\&G Program of the University Medical Center of the University of Groningen. In the first part of the training, the theoretical body of thought behind the G\&G interventions is explained. In the second part, the intervention-manual is practiced through modeling and role-play. At the end of the training the professionals are being instructed on the content of the G\&G implementation toolkit, which is developed by the G\&G project team, and which offers a variety of materials supporting their implementation activities (e.g. PR materials, press release examples, brochures, etc.). The trained professionals are also informed about various facilitating activities offered by the G\&G project team (i.e. website, annual work conference, and site visits).

\section{Study setting}

The study is performed in health and social care organizations for older adults in the northern part of the Netherlands. Since 2007 municipal authorities are responsible for supervision and execution of the Social Support Act, which prescribes that vulnerable older adults and other vulnerable citizens need to be supported to recapture or maintain their ability to manage their own well-being. In consultation with the management of health and social care organizations, municipal policies are determined and available resources are allocated. Each municipality has one or more health and social care organizations that employ a variety of professionals. Professionals can either be social workers employed in welfare organizations or health professionals employed in home care organizations, providing both physical and psychosocial care to their clients. They can also be activity leaders employed in retirement homes, striving to empower residents and 
older adults living in sheltered accommodations next to the home.

\section{Study sample}

The study sample consists of actors at four different hierarchical stakeholder levels (i.e. target group, professionals, organizations and financial-political context) as depicted in Figure 2. Therefore, there are four groups of informants.

The first group (i.e. the target group) consists of the older adults at risk of becoming vulnerable. The second group are the trained professionals, who deliver the G\&G interventions. The third group consists of the managers of the participating organizations. When organizations have multiple management-layers, the manager who is closest to the work floor will be invited to act as key-informant. The fourth and final group consists of key informants at the level of the financial-political context. These are local policymakers who are well informed on the execution of the Social Support Act. They will be invited to act as informants for our study.

Based on experiences from an earlier pilot-implementation of the G\&G interventions, it is feasible to include at least 15 new organizations in a period of 12 months [35]. Counting with 15 organizations, the sample size at the organizational and financial-political level will be 15 managers and 15 local policymakers. With a minimum of two G\&G professionals per organization, the sample size at the professional level will be at least $30 \mathrm{G} \& \mathrm{G}$ professionals.

With respect to the reach of the target group, concrete performance goals are communicated with the G\&G professionals. Each $G \& G$ coach is expected to deliver the G\&G home visits to at least three older adults (15 G\&G coaches $\times 3$ home-visits $\times 1$ participant $=45$ participants) and each pair of G\&G teachers is expected to deliver at least three group courses, with an average of ten older adults per course (15 G\&G teacher pairs $\times 3$ group-courses $\times 10$ participants $=450$ participants). In the 2-year period of the data-collection, the sample size at the level of the target group will thus amount to approximately 495 older adults. Taking into account a drop-out rate of $8 \%$ [36] a maximum number of 400 older adults participating in the G\&G interventions is expected to be feasible.

\section{Procedure and measures}

The degree of implementation success will be assessed per organization, and is being operationalized by four parameters: use (yes/no), pace (time to initial use), performance (extent of use) and prolongation (intention to

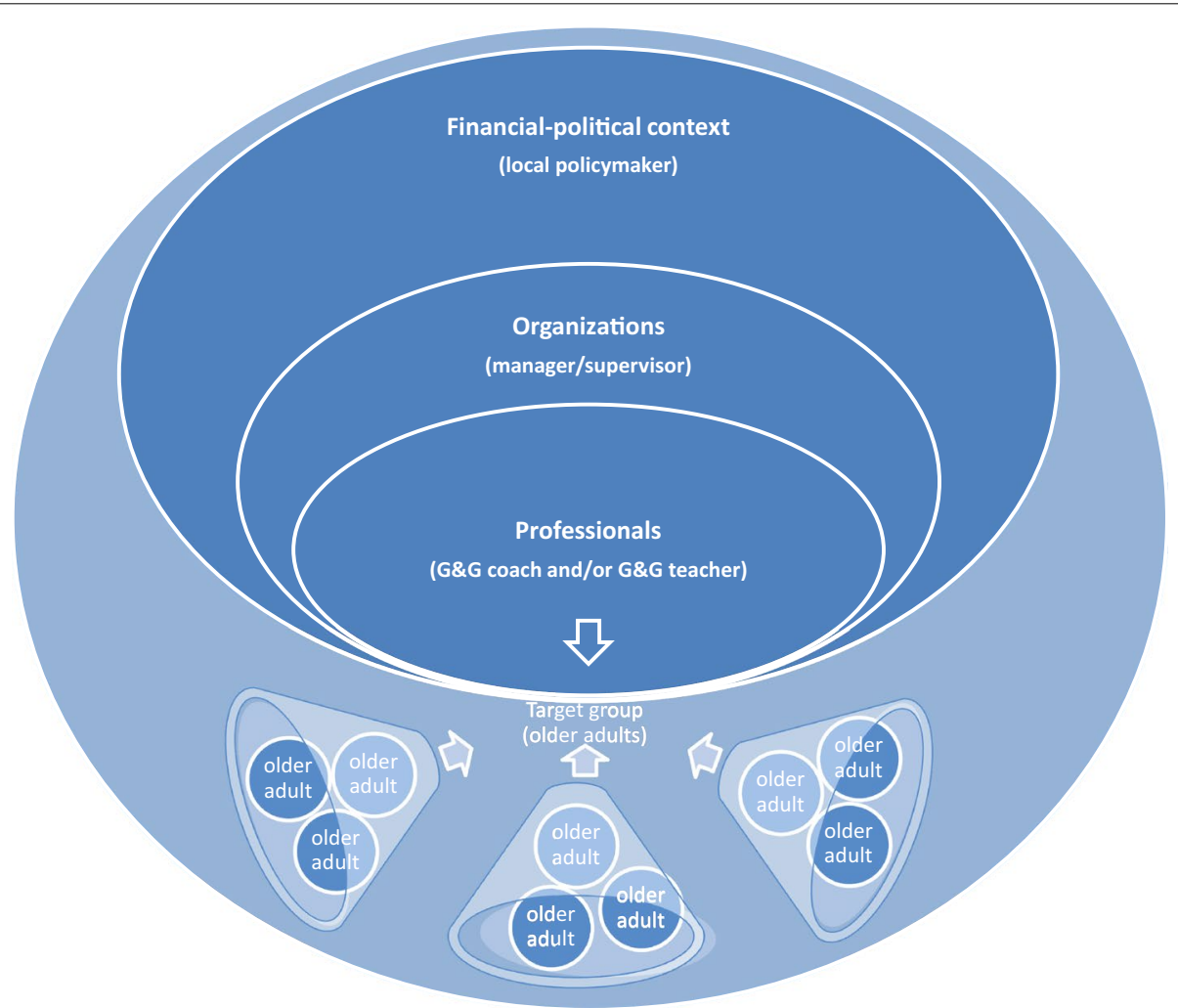

Figure 2 Hierarchical stakeholder levels. 
continue use beyond the timeframe of the study). The rationale behind the selection of these four parameters is that they assess the transitions between the three consecutive implementation stages in the Fleuren model (see Figure 1). The 'use' parameter measures the transition of organizations from adoption to implementation and the 'prolongation' parameter measures the transition from implementation to continuation. Next, we expect the 'pace' and 'performance' parameter to add to the explanation of both transitions.

Use, pace and performance can be easily assessed and with very high validity, because the actual performance of all organizations regarding the use of the G\&G interventions will be monitored continuously throughout the study by the project team. The fourth and final parameter (i.e. intention to continue use of the G\&G interventions beyond the time frame of the study) can necessarily only be measured as an estimation of the relevant actors. The intention of each professional, each manager, and each financial-political key informant, to continue the use of the G\&G interventions beyond the timeframe of the study (i.e. prolongation) is operationalized with a single question with four answer categories ranging from (0) no intention to (3) strong intention. This question will be asked at the final measurement point of the study.

The facilitating and impeding factors that possibly affect the use of the G\&G interventions are being measured at multiple measurement moments, simultaneously at the four hierarchical levels (i.e. target group, professionals, organizations and financial-political context). The content of the questionnaires varies somewhat per measurement point, because some factors only apply to the specific stage the organizations are in. For example, "ownership" is only applicable when users move from the adoption to the (initial) implementation stage, while "observability of effects" is applicable only when users move from the implementation to the continuation stage. In the following a brief outline of the measures is given. Details on the specific measurement moments, and at what point in time we measure which factors, are described in Additional File 3.

\section{Target group}

The impeding and facilitating factors at the level of the target group will be measured via the perception of the G\&G professionals. This indirect way is necessary, because the factors at this level relate to possible participants, not to actual participants of the intervention. A sample of all possible participants (in the population) is hard to delineate. Therefore, the professionals will be asked to answer the questions of the target group level. The professionals will well be able to give an estimation of the impeding and facilitating factors that possibly play a role for older adults to participate (or not) in the G\&G interventions, due to their large experience with the target group. The predefined factors of the theoretical framework at the level of the target group are thus translated into questions to be answered by the professionals. For example, the factor "awareness of benefits" is translated into the question: "Do you think older adults understand the benefits of participating in the G\&G interventions?" The questions contain six answer categories ranging from (0) 'not at all' to (5) 'completely'.

\section{Professionals}

The impeding and facilitating factors at the level of the professionals will be assessed by means of digital questionnaires. All professionals who have been trained and certified as G\&G coach and/or G\&G teacher will be invited to fill out the questionnaire. The predefined factors of the theoretical framework at the level of the professionals are translated into one or more questions per factor. For example, the factor "ownership" is translated into the question "To what extent do you feel responsible for G\&G intervention start-up?" Each question contains six answer categories ranging from (0) 'not at all' to (5) 'completely'.

\section{Organizations}

The impeding and facilitating factors at the level of the organizations will be measured by means of a telephone interview with the managers. The predefined factors of the theoretical framework at the level of the organization are translated into one or more questions per factor. For example, the factor "staff capacity" is translated into the question "Is your staff capacity sufficient to spend time on integrating the G\&G interventions in routine practice?" The questions contain six answer categories ranging from (0) 'not at all' to (5) 'completely'.

\section{Financial-political context}

The impeding and facilitating factors at the level of the financial-political context will also be measured by means of a telephone interview with a strategic or financial local policymaker. The predefined factors of the theoretical framework at this level are translated into one or more questions per factor. For example, the factor "added value" is translated into the question "To what extent do you think that the G\&G interventions add something to the existing services for older people in your community?" The questions contain six answer categories ranging from (0) 'not at all' to (5) 'completely'.

\section{Data analysis}

All data will be imported in SPSS statistics 20. Descriptive analysis will be used to characterize use, time to initial use (pace), extent of use (performance) and the 
intention to continue use beyond the timeframe of the study (prolongation). Data collected at the four stakeholder levels will be merged and aggregated. Intra-class correlations will be calculated to assess the reliability of individual data aggregated at group levels in hierarchical models (i.e. professionals nested in organizations). The relevance of applying multilevel modeling to the data will be assessed by testing an unconditional or null model in which no predictors are specified. Only when significant variations in the dependent variables are present across organizations or municipalities, multilevel regression modeling will be applied. If no significant variation in use, pace, performance or prolongation is found across organizations or municipalities, we confine to single-level modeling techniques.

\section{Discussion}

The aim of the study described in this protocol is to identify the determinants of successful implementation of the G\&G interventions. In this study, health and social care organizations are considered to be part of multilevel functional systems [37], in which factors at different hierarchical levels can impede or facilitate the actual use of the G\&G interventions. By assessing the unique contribution of target group, professional, organizational and financial-political context factors, as well as the complex interplay between these factors, results are expected to be of added value to the current scientific knowledge on barriers and facilitators to EBP-use.

The study has several strengths. The first is that implementation success in this study is not only assessed by the parameter use (yes/no), but it is also specified in three other indicators of use, namely: pace, performance and prolongation. This approach yields a much more specified insight in the various aspects of implementation success. Second, the possible factors that are considered in this study are theoretically supported by the Fleuren model, which provides a solid basis and prevents an ad hoc selection of possible factors. Finally, the analyses of the complex interplay between factors at different hierarchical stakeholder levels are executed with advanced multilevel modeling techniques. This is the optimal way of doing justice to the multi-layered nature of reality in implementation processes.

In conclusion, globally [38] and nationally [39] there is momentum to invest in EBPs that support aging individuals to live full, enriching and productive lives for as long and as much as possible. The G\&G interventions are an example of such EBPs. They have been designed and tested in the last decade. Now it is time to increase our understanding of how to transport them to health and social care settings in a sustainable way. Identifying the determinants of successful implementation of the G\&G interventions will also contribute to the development of ecologically valid implementation strategies of the G\&G interventions and comparable new evidence-based practices.

\section{Additional files}

Additional file 1: Two-step procedure constructing theoretical framework.

Additional file 2: Time plan G\&G-implementation study.

Additional file 3: Factors, time points and number of items per stakeholder level.

\section{Abbreviations}

G\&G: GRIP\&GLEAM [Dutch: GRIP\&GLANS]; GRIP stands for the ability to adapt and self-manage. GLEAM stands for well-being and the feeling that life is good and worth living; EBP: Evidence Based Practice; a practice that has been established as effective through scientific research according to a set of explicit criteria.

\section{Authors' contributions}

DK conducts the study and drafted and edited the final manuscript. NS conceived of the study, obtained funding, provides leadership and coordination in the conduct of the study. MMG and NS helped to draft the manuscript. RS and SAR participated in critically appraising and revising the intellectual content of the manuscript. All authors agree to be held accountable for all aspects of the work. All authors read and approved the final manuscript.

\section{Author details \\ ${ }^{1}$ Department of Health Sciences, University Medical Center Groningen, Uni- versity of Groningen, Groningen, The Netherlands. ${ }^{2}$ Department of Psychol- ogy, Health and Technology, University of Twente, Enschede, The Netherlands. \\ ${ }^{3}$ Faculty of Behavioural and Social Sciences, University of Groningen, Gronin- gen, The Netherlands.}

\section{Acknowledgements}

This study is funded by ZonMw, the Netherlands Organization for Health Research and Development. Grant number: 313010401 in the National Care for the Elderly Program.

\section{Compliance with ethical guidelines}

\section{Competing interests}

The authors declare that they have no competing interests. NS designed the $G \& G$ interventions, but has no financial interest in the extent to which they are used in health and social care settings.

Received: 10 December 2013 Accepted: 30 June 2015

Published online: 07 July 2015

\section{References}

1. Christensen K, Doblhammer G, Rau R, Vaupel JW (2009) Ageing populations: the challenges ahead. Lancet 374(9696):1196-1208

2. Huber M, Knottnerus JA, Green L, Horst H, Jadad AR, Kromhout D et al (2011) How should we define health? BMJ 343:d4163. doi:10.1136/bmj.d4163

3. Victor CR, Scambler SJ, Bowling A, Bond J (2005) The prevalence of, and risk factors for, loneliness in later life: a survey of older people in Great Britain. Ageing Soc 25(3):357-375

4. Valtorta N, Hanratty B (2012) Loneliness, isolation and the health of older adults: do we need a new research agenda? JRSM 105(12):518-522

5. Nicholson NR (2012) A review of social isolation: an important but underassessed condition in older adults. J Prim Prev 33(2-3):137-152 
6. Beekman A, Copeland J, Prince MJ (1999) Review of community prevalence of depression in later life. Br J Psychiatry 174(4):307-311

7. Zantinge EM, van der Wilk EA, van Wieren S, Schoemaker CG (2011) Gezond ouder worden in Nederland. RIVM, Bilthoven

8. Wilson DM, Harris A, Hollis V, Mohankumar D (2011) Upstream thinking and health promotion planning for older adults at risk of social isolation. Int J Older People Nurs 6(4):282-288

9. Schuurmans $\mathrm{H}$ (2004) Promoting well-being in frail elderly people: theory and intervention. PhD thesis, Groningen University, Groningen

10. Kremers IP, Steverink N, Albersnagel FA, Slaets JP (2006) Improved selfmanagement ability and well-being in older women after a short group intervention. Aging Ment Health 10(5):476-484

11. Steverink N, Lindenberg S, Slaets JP (2005) How to understand and improve older people's self-management of well-being. Eur J Ageing 2:235-244

12. Zwet R, Beneken Genaamd Kolmer D, Schalk R (2011) Op weg naar een interactieve benadering van evidence-based werken in de sociale sector in Nederland. J Soc Interv Theory Prac 20(4):62-78

13. Mullen EJ, Bledsoe SE, Bellamy JL (2008) Implementing evidence-based social work practice. Res Soc Work Prac 18(4):325-338

14. Proctor EK, Landsverk J, Aarons G, Chambers D, Glisson C, Mittman B (2009) Implementation research in mental health services: an emerging science with conceptual, methodological, and training challenges. Adm Policy Ment Health 36(1):24-34

15. McGlynn EA, Asch SM, Adams J, Keesey J, Hicks J, DeCristofaro A et al (2003) The quality of health care delivered to adults in the United States. N Engl J Med 348(26):2635-2645

16. Palinkas LA, Soydan H (2012) New horizons of translational research and research translation in social work. Res Soc Work Prac 22(1):85-92

17. Mclntosh K, Horner RH, Sugai G (2009) Sustainability of systems-level evidence-based practices in schools: Current knowledge and future directions. In: Handbook of positive behavior support. Springer, pp 327-352

18. Grol R, Wensing M (2004) What drives change? Barriers to and incentives for achieving evidence-based practice. Med J Aust 180(6 Suppl):S57-S60

19. Fleuren M, Wiefferink K, Paulussen T (2004) Determinants of innovation within health care organizations: literature review and Delphi study. Int J Qual Health Care 16(2):107-123

20. Greenhalgh T, Robert G, Macfarlane F, Bate P, Kyriakidou O (2004) Diffusion of innovations in service organizations: systematic review and recommendations. Milbank Q 82(4):581-629

21. Fixsen DL, Naoom SF, Blase KA, Friedman RM, Wallace F (2005) Implementation research: a synthesis of the literature. Tampa, FL: University of South Florida, Louis de la Parte Florida Mental Health Institute, The National Implementation Research Network (FMHI Publication \#231)

22. Stith S, Pruitt I, Dees J, Fronce M, Green N, Som A et al (2006) Implementing community-based prevention programming: A review of the literature. J Prim Prev 27(6):599-617

23. Durlak JA, DuPre EP (2008) Implementation matters: a review of research on the influence of implementation on program outcomes and the factors affecting implementation. Am J Community Psychol 41(3-4):327-350

24. Thomas R, Zimmer-Gembeck MJ, Chaffin M (2014) Practitioners'views and use of evidence-based treatment: positive attitudes but missed opportunities in children's services. Adm Policy Ment Health 41:368-378. doi:10.1007/s10488-013-0471-y
25. Sanders MR, Prinz RJ, Shapiro CJ (2009) Predicting utilization of evidencebased parenting interventions with organizational, service-provider and client variables. Adm Policy Ment Health 36(2):133-143

26. Asgary-Eden V, Lee CM (2011) So now we've picked an evidence-based program, what's next? Perspectives of service providers and administrators. Prof Psychol Res Prac 42(2):169

27. Kilbourne AM, Neumann MS, Pincus HA, Bauer MS, Stall R (2007) Implementing evidence-based interventions in health care: application of the replicating effective programs framework. Implement Sci 2:42

28. Feldstein AC, Glasgow RE (2008) A practical, robust implementation and sustainability model (PRISM) for integrating research findings into practice. Jt Comm J Qual Patient Saf 34(4):228-243

29. Wandersman A, Duffy J, Flaspohler P, Noonan R, Lubell K, Stillman L et al (2008) Bridging the gap between prevention research and practice: the interactive systems framework for dissemination and implementation. Am J Community Psychol 41(3-4):171-181

30. Mendel P, Meredith LS, Schoenbaum M, Sherbourne CD, Wells KB (2008) Interventions in organizational and community context: a framework for building evidence on dissemination and implementation in health services research. Adm Policy Ment Health 35(1-2):21-37

31. Damschroder LJ, Aron DC, Keith RE, Kirsh SR, Alexander JA, Lowery JC (2009) Fostering implementation of health services research findings into practice: a consolidated framework for advancing implementation science. Implement Sci 4:50

32. Stetler CB, Damschroder LJ, Helfrich CD, Hagedorn HJ (2011) A guide for applying a revised version of the PARIHS framework for implementation. Implement Sci 6:99

33. Aarons GA, Hurlburt M, Horwitz SM (2011) Advancing a conceptual model of evidence-based practice implementation in public service sectors. Adm Policy Ment Health 38(1):4-23

34. Fleuren M, Wiefferink K, Paulussen T (2010) Checklist determinanten van innovaties in gezondheidszorgorganisaties. TSG 88(2):51-54

35. Elzen H, Steverink N (2009) Een landelijk implementatie-project m.b.t. de GRIP- en GLANS-cursussen: een zelfmanagement-cursusaanbod voor ouderen. Eindrapport. (national implementation project of the GRIP and GLANS courses: a self-management of well-being program for older adults; internal report): Groningen: University of Groningen, University Medical Center Groningen

36. Hunt C, Andrews G (1992) Drop-out rate as a performance indicator in psychotherapy. Acta Psychiatr Scand 85(4):275-278

37. Fixsen DL, Blase KA (2009) Implementation: The missing link between research and practice. NIRN Implement Brief 1

38. Good health adds life to years. Global brief for world health day 2012 (2012). http://www.who.int/ageing/publications/whd2012_global_brief/ en/. Accessed 3 July 2015

39. The National Care for the Elderly Programme (2015). http://www.beteroud.nl/ouderen/dutch-national-care-programme-for-the-elderly.html. Accessed 3 July 2015

\section{Submit your next manuscript to BioMed Central and take full advantage of:}

- Convenient online submission

- Thorough peer review

- No space constraints or color figure charges

- Immediate publication on acceptance

- Inclusion in PubMed, CAS, Scopus and Google Scholar

- Research which is freely available for redistribution

Submit your manuscript at

www.biomedcentral.com/submit
C Biomed Central 